\title{
La constitutionnalisation des droits de minorités au Cameroun: Usages politiques du droit et phobie du séparatisme
}

\author{
Par Jean Njoya, Yaoundé
}

Le Cameroun est entré dans le concert des nations avec toutes les difficultés liées à la mise en place de ses institutions politiques. Son originalité tient au bilinguisme hérité des administrations coloniales française et anglaise. Il s'est posé un problème de convivialité entre les deux communautés aux systèmes culturels diamétralement opposés. La minorité anglophone, recevait mal le Droit public français fondé pour l'essentiel sur la puissance publique assortie de toutes les prérogatives exhortantes de droit commun. Par conséquent, les anglophones faisaient valoir la vertu du Common law plus protecteur des droits des citoyens.

Dans ce contexte on eut pu redouter un radicalisme irréductible dans la perception philosophique de l'orientation que les "entrepreneurs politiques" du nouvel État allaient donner aux institutions politiques. Loin s'en est fallu. Le processus s'est opéré avec une "sage" lenteur de la forme fédérale de l'Etat à la forme unitaire.

Mais, faut-il le rappeler, les premiers dirigeants Camerounais ont largement fait usage d'une interprétation personnelle des lacunes et des ambiguïtés de la constitution. Toute équivoque, tout malentendu et toute ambiguïté étaient levés par un exposé de motifs soutenu par les préoccupations unitaristes. Une telle attitude s'imposait d'ailleurs aux dirigeants camerounais. Et sur ce dernier trait, il n'est pas superflu de souligner que le Cameroun par sa complexité sociologique se présente somme toute comme un véritable bestiaire des fractures géopolitiques ${ }^{1}$. Fracture macropolitique entre la communauté francophone et la communauté anglophone et micropolitique entre les 230 groupes ethniques qui badigeonnent le paysage politique camerounais. La vigueur de ces particularismes imposait donc au constituant camerounais une option centralisatrice plus commode et plus apte à gommer toutes les aspérités centrifuges des replis identitaires.

J. Njoya, Cameroun, bestiaire géopolitique des fractures sociales, Université Yaoundé II, 1997, pp. 1 - 22. 
A la lecture des constitutions du $1^{\text {er }}$ Septembre 1961, du 2 Juin 1972 et plus récemment celle du 18 Janvier 1996, il se dégage une impression générale: C'est la charge de l'idée de l'unité nationale sur la mise en place des institutions politiques camerounaises. ${ }^{2}$

La constitution du $1^{\text {er }}$ Septembre 1961 a instauré un fédéralisme de convenance avec une répartition des compétences relativement conforme au fédéralisme classique et sur lequel pesait une véritable "chape de plomb" centralisatrice. Si le clivage anglophone/francophone a été pris en compte dans cet encadrement juridique, on y ressentait toutefois la pesanteur de l'attraction centripète. C'était en réalité une amorce feinte vers l'Etat unitaire avec un grignotage progressif du fédéralisme originel.

Celle du 2 Juin 1972, est l'aboutissement d'un long processus dont le fédéralisme monocaméral du 1er Septembre 1961 annonçait déjà les signes précurseurs. L'Etat unitaire qui en a résulté a installé dans la conscience collective une "pseudo-convivialité » ${ }^{3}$ entre la communauté francophone et la communauté anglophone. Depuis lors, elles entretiennent entre elles un climat de suspicion qui a généré lors de l'instauration du multipartisme de nouvelles formes de replis identitaires confinant parfois le sécessionniste.

L'acte constitutionnel du 18 Janvier 1996 n'a pas tranché avec cette logique unitariste. Il a semblé d'ailleurs la conforter si on en juge par les précautions politiques prises par le chef de l'Etat pour étouffer la cristallisation des clivages entre les deux communautés. Envisagée dans la perspective de la prise en compte de nouveaux facteurs de la subversion sociale née du pluralisme politique, les constituants du 18 Janvier 1996 ont été amené à pénétrer au cœur de l'une des questions les plus troublantes de notre temps: celles des minorités, et des autochtones. Il s'agit des notions aux contours du reste flous et la manière dont le constituant s'en est servi traduit le peu d'intérêt accordé à la précision de ces concepts. Pour montrer comment le «nous » ${ }^{4}$ se constitutionnalise dans le cadre camerounais, ces textes serviront d'appui analytique.

Sur cette base, deux idées principales nous paraissent préoccupantes: d'abord, la mise en place des institutions politiques et l'illusion de la prise en compte du biculturalisme. Ensuite, la manifestation d'une nette tendance centralisatrice dans les textes postérieurs.

K. A. Kontchou, Idéologie et institutions politiques: l'impact de l'idée de l'unité nationale sur les institutions politiques de l'Etat camerounais, dans G. Conac (ed.), Dynamiques et finalités des droits africains, Paris, Economica, 1980, p. 442.

3 Nkoum-Me-Ntseny, Dynamique de positionnement anglophone et libéralisation politique au Cameroun, R.C.S.P., Vol N¹, Yaoundé, 1996, p. 70.

4

L. Sindjoun, Identité et révision constitutionnelle du 18 Janvier 1996: Comment constitutionnalise-t-on le "nous" au Cameroun dans l'Etat Post-unitaire, R.C.S.P. 1996, N 1 p. 10. 


\section{L'acte constitutionnel du 1er septembre 1961: L'illusion de la prise en compte de l'identité bicommunautaire}

Le professeur Kontchou Kouemegni fait une observation qui résume l'idée qui a toujours animé le constituant camerounais: «L'état unitaire de 1960 écrit-il fut à peine institué que s'y substituait la fédération qui, dix ans plus tard devait céder la place à un État unitaire centralisé $»^{5}$ En effet, le caractère imprécis de l'Etat camerounais est apparu dès les premières heures de son indépendance. L'institution d'un État unitaire permanent eut été peu commode dans un contexte de biculturalisme. Très tôt s'est manifestée la volonté d'une prise en compte du legs colonial Franco-Britannique. En fait, il fallait traduire en profondeur les préoccupations existentielles fondamentales du peuple Camerounais à un moment donné de son histoire ; a-t-on encore besoin de signaler que la constitution du 4 Mars 1960 tirait son caractère éphémère de cet état de choses. La promotion d'un fédéralisme centralisateur s'est avérée comme un replâtrage constitutionnel pour étouffer les revendications identitaires qui avaient culminé lors de la conférence constitutionnelle de Foumban les 17, 18, 19, 20, 21 Juillet 1961. Cet arrangement était en réalité une espèce de cote mal taillée au grand dam du fédéralisme classique.

\subsection{Un fédéralisme de convenance: une cote mal taillée}

Dans l'énorme littérature consacrée à la nature de l'Etat fédéral du $1^{\text {er }}$ Septembre 1961 ,on rencontre tous les points de vue. La tendance dominante relève le caractère insolite du fédéralisme Camerounais au vue des expériences classiques (U.S.A., U.R.S.S.). Ce constat repose pour l'essentiel sur la nature trop centralisatrice de l'Etat et sur l'institution du monocaméralisme. En réalité, si la répartition des compétences entre le pouvoir central et les États fédérés était relativement conforme au fédéralisme classique, on note toutefois la prégnance d'une véritable "chape de plomb" centralisatrice sur les institutions étatiques de 1961.

\subsubsection{Une répartition des compétences relativement conforme au fédéralisme classique}

Les fédéralistes font généralement valoir leur intention de décongestionner ou mieux de décentraliser à suffisance l'Etat pour assurer l'administration des administrés et l'autonomie des composantes organiques de la périphérie. Qui plus est, ils évoquent la nécessité d'une conciliation entre l'unité et la diversité de l'Etat. ${ }^{6}$

\footnotetext{
A. d. Tocqueville, De la démocratie en Amérique, Paris, 1835.
} 
On ne "gobe" pas les bonnes intentions, on les passe au crible de la réalité. La vérité est que tous États fédéraux manifestent à des degrés divers une nette tendance à un renforcement progressif du pouvoir central au détriment des États fédérés. ${ }^{7}$ A la dynamique centrifuge des États fédérés, le pouvoir central oppose insidieusement une force d'attraction centripète. Cette centralisation s'est avérée outrancière dans le compromis constitutionnel du $1^{\mathrm{er}}$ septembre 1961.

\subsubsection{La consistance des compétences fédérales: des attributions immédiates et progressives}

La constitution du $1^{\text {er }}$ septembre 1961 avait toutes les allures d'une constitution-programme $^{8}$; car, elle devait traduire la marche progressive vers la réalisation de l'unité nationale. ${ }^{9}$ Pour cette raison, les compétences de l'Etat fédéral allaient se développer tel l'hydre de Lerne.

\section{Les compétences immédiates de l'Etat fédéral}

L'article 5 les résume pour l'essentiel en cinq rubriques. Elles se rattachent aux attributions de souveraineté et aux matières sensibles. Il s'agit des affaires internationales (immigration, défense, relations commerciales, statut des étrangers, sûreté intérieure et extérieure, assistance technique, relations financières internationales).

La deuxième rubrique comportait la culture (enseignement supérieur, recherche scientifique, information, couverture géographique, économique, plan, statistiques, orientation économique, mines et recherche géologique). Viennent ensuite les affaires financières (organisation et contrôle de crédit, l'établissement du Budget fédéral, les impôts et les recettes, le régime monétaire).

D'autres matières non moins importantes s'ajoutent à cette énumération (organisation et fonctionnement de la Cour fédérale de justice, statut des magistrats), le statut des personnes (nationalité, conflit des lois), le statut de la fonction publique fédérale, les limites territoriales des États fédérés.

A l'évidence, il s'agit des compétences immédiates qui devaient aller croissantes en fonction des exigences conjoncturelles de l'intégration nationale.

Voir N. Kemph / M. F. Toinet, La fin du fédéralisme aux U.S.A., R.F.S.P., 1980, pp. 735 et suivants.

8 Voir G. Burdeau, Traité de Science Politique, Paris LGDJ, 1969 T.IV p.131

9

Discours d'Ahmadou Ahidjo le 18-02-1958. 


\section{Les compétences potentielles des autorités fédérales}

Il s'agit de véritables attributions pour lesquelles l'intervention du pouvoir central était conditionnée. L'article 6 en énumère les plus importantes: le régime des libertés publiques, le statut des personnes et des biens, le régime des obligations et contrat en matière civile et commerciale, de l'organisation judiciaire, du droit pénal, du transport d'intérêt général, des ports, de l'administration pénitentiaire, de la législation domaniale, du travail, de la santé publique, de l'enseignement secondaire et technique, de l'organisation administrative et des poids et mesures.

La compétence des États fédérés dans ces matières était conditionnée par la consultation d'une commission fédérale désignée par le Chef de l'Etat. On peut même dire que l'avis de cette commission était un avis conforme, puisque son contrôle s'étendait à la fois sur la régularité et l'opportunité. La célérité avec laquelle ces matières ont été aussitôt transférées à l'Etat fédéral après la réunification était déjà un signe annonciateur de la pesanteur des forces centripètes. Agissant ainsi sous le bénéfice d'inventaire, les États fédérés allaient se voir confier des matières fort résiduelles.

\subsubsection{Le caractère sommaire des compétences fédérées dans la constitution de septembre 1961}

La formulation de l'article 38 incline à une énumération par déduction. Cette disposition paraît à la fois elliptique et saturée de silences. En effet, elle énonçait que: «les matières autres que celles énumérées aux articles 5 et 6 ou mentionnées dans la présente constitution comme devant faire l'objet d'une loi fédérale, relèvent exclusivement de la compétence des États fédérés ». On peut imaginer au regard de la pratique des États fédérés qu'il s'agissait de l'agriculture, de la forêt, des coopératives, de l'élevage, des travaux publics, de l'administration locale, de l'enseignement primaire, du droit coutumier.

Dans une telle répartition fortement inégalitaire, l'affirmation constitutionnelle de la forme fédérale devenait une clause de style pour masquer de nettes tendances centralisatrices. Dans les faits, certains auteurs entrevoyaient déjà la fin inéluctable de la fédération ${ }^{10}$ à laquelle faisait constamment allusion le président Ahidjo non sans révéler son obsession pour l'unité nationale. 11

Cette centralisation outrancière des institutions se trouvait accentuée par l'institution d'une chambre unique qui rendait d'avantage insolite le fédéralisme camerounais. Le monocaméralisme exerçait une sorte d'attraction centripète sur les institutions fédérées. Ce d'autant 
que l'inexistence d'un sénat faisait fondre les représentations des États fédérés dans une chambre unique.

\subsection{La "chape de plomb "centralisatrice: La pesanteur de l'attraction centripète}

Dans le système fédéral, l'autonomie constitutionnelle des États fédérés est garantie par l'existence d'une seconde chambre qui assure leur représentation équitable. Le fédéralisme institué par la constitution de 1961 s'est opéré par association. L'entrée du Cameroun Occidental dans la fédération n'entraînait pas une fusion, mais simplement une agrégation à une autre entité unie moins intimement.

Le monocaméralisme institué par la constitution de 1961 conforte le caractère éminemment spécifique du fédéralisme camerounais, alors même que l'expérience de presque tous les pays fédéraux du monde consacre le dualisme du parlement qui «explique tant l'existence du sénat face à la chambre des représentants que celle du soviet des nationalités face au soviet de l'union dans l'ancienne U.R.S.S. » ${ }^{12}$. Pour obtenir la mise en sourdine des revendications identitaires de la communauté anglophone, le constituant avait trouvé une parade convenable.

Ainsi, les articles 18 et 47 exigeaient deux sous-majorités pour une loi fédérale en seconde lecture ou une loi de révision constitutionnelle. ${ }^{13}$ Ces dispositions eussent pu satisfaire les exigences de l'égalité entre les États fédérés si le constituant n'avait pas laissé l'initiative de la seconde lecture au président de la fédération. Et même, la suggestion purement consultative des premiers ministres des États fédérés faisait du président de la République le seul juge de l'opportunité de l'intervention.

Le déséquilibre entre les communautés anglophone et francophone pouvait se lire à travers les coteries politiques qui ont précédé la consultation du $1^{\text {er }}$ septembre 1961.Lors de la conférence constitutionnelle de Foumban tenue les 17,18,19 20, 21 juillet, le président AHIDJO fit une déclaration confuse qui suscita un étonnant acquiescement de la délégation anglophone. Il précisait en effet «qu'il ne considérait que les propositions présentées sous la forme de modification à la constitution de $1960 »{ }^{14}$. Il faut avouer que les représentants anglophones à Foumban avaient l'échine souple et ont même été complaisants jusqu'à la servilité.

K. A. Kontchou, Les pouvoirs du Chef de l'Etat camerounais, Mémoire de Licence, Université de Yaoundé, 1969, p. 70.

13

A savoir celles des groupes des députés élus dans chacun des deux Etats fédérés.

14

J. Benjamin, Les Camerounais occidentaux, la minorité dans un Etat bicommunautaire, Montreal, Les presses de l'Université de Montreal, 1972, p. 112. 
Dans une déclaration sémantiquement chargée de révérence, le premier ministre John Ngu Foncha révèle au président Ahidjo dans un ton que Jacques Benjamin qualifie de « suppliant », la modestie des ses propositions: «Depuis trois jours, nous avons étudié la position que votre excellence nous a présentée. Nous y avons trouvé plusieurs points d'entente (...). Je veux assurer son excellence que les suggestions que nous avons faites lors de ces discussions ne sont pas parfaites (...). Les recommandations que nous avons formulées proviennent du sentiment de fraternité envers la République du Cameroun ${ }^{15}$.

Cette attitude avait naturellement accordé des coudées plus franches au Président Ahidjo qui a réalisé avec ruse un véritable "exploit" constitutionnel à la Pyrhus. Le droit positif camerounais ne saurait aménager une confortable hospitalité à l'argument du "troc dolosif" que mettent en avance certains auteurs ${ }^{16}$. Cette expression a peut-être une signification dans l'univers passionné de la politique. Juridiquement, une telle turpitude est inallégable pour obtenir retrait d'un acte pour lequel l'on a lucidement et activement participé à la signature. Ce "rattrapage politique" s'ouvre donc sur un non-lieu.

Ainsi, la constitution du $1^{\text {er }}$ septembre 1961 a été négociée et mise en place dans une atmosphère qui ne faisait plus mystère de l'obsession unitariste du Président Ahidjo. Ce dernier allait réaliser après la parenthèse fédérale son chant de cygne par un grignotage progressif des aspérités fédéralistes. C'était l'amorce feinte vers l'Etat unitaire.

\subsection{L'amorce feinte vers l'Etat unitaire: le grignotage progressif des aspérités fédéralistes ou la lente corrosion de l'identité politique anglophone}

L'Etat fédéral fut finalement un tremplin pour la réalisation d'un projet que le président Ahidjo tenait encore dans la langue de bois. Pour ce faire il fallait conditionner l'atmosphère politique avant d'assener le "coup de grâce" le 2 juin 1972.

\subsubsection{Les préludes politiques: la promotion du parti unifié}

L'identité politique anglophone s'est noyée dans une confusion générale apparemment entretenue volontiers par le président Ahidjo. Déjà après son élection le 5 mai 1960, il lança un appel pour la formation d'un parti unifié autour de l'Union Camerounaise. Il ne s'agissait pas bien évidemment d'une fédération des partis au sein de laquelle chaque formation allait conserver son identité propre. ${ }^{17} \mathrm{~S}$ 'en suivra une avalanche de ralliements 
des partis modérés non sans provoquer des dissensions au sein des formations politiques radicales. Mr Charles Assale (Premier Ministre) fut le premier à jeter le manche après la cognée. La dissolution du Mouvement d'Action Nationale en février 1961 répondait à cet appel d'unité. Depuis lors, la mécanique de la fusion s'était avérée difficile à être enrayée. Les "progressistes" du Front Populaire Uni, l'Union Nationale des Associations Traditionnelles du Cameroun fusionnèrent au sein de l'U.C. à partir de 1961. Les leaders qui cherchèrent à se raviser après cette phagocytose furent arrêtés et reconnus "coupables d'incitation à la haine contre le gouvernement". La sévérité de la condamnation prouve que le Chef de l'Etat était résolument passé de la négociation à l'intimidation. ${ }^{18}$

Au début de l'année 1963, l'opposition parlementaire était réduite à 7 députés. Une présence bien figurative si on songe qu'en 1960 l'opposition avait toute sa raison d'être (avec 48 députés, elle talonnait l'Union Camerounaise de 4 députés d'écart). Curieusement, le 25 Août 1965 sans qu'aucune pression n'ait été exercée sur eux, les leaders anglophones annoncèrent leur intention de fusionner. La naissance de l'Union Nationale Camerounaise le $1^{\text {er }}$ septembre 1966 «consacrait ainsi la disparition des anciens partis et la suprématie de l'Union Camerounaise »" ${ }^{19}$. Si l'aile anglophone ne s'est pas laissée enfermer dans la torpeur de la vie politique du Cameroun Oriental ${ }^{20}$, on peut tout de même affirmer que le "suicide politique" était consommé et le président Ahidjo devait traduire la portée démesurément significative de ces ralliements au premier congrès de l’U.N.C.

« (...) Nous voilà réunis en congrès pour consacrer un événement qui aura dans l'histoire politique de notre pays, j'ose dire l'histoire politique de l'Afrique, une signification d'une exceptionnelle portée (...). » ${ }^{21}$ La rétribution des dividendes politiques de cette collaboration allait être naturellement sélective: les alliés du parti gouvernemental furent hissés au Capitole condamnant ainsi à la Roche Tarpienne les plus irréductibles des opposants. Avec délectation le Chef de l'Etat accueillait messieurs Mayi Matip et Inack Njocki (ancien leader de l'U.P.C.) en 1968 avec tous les honneurs que justifiait leur long combat au sein du parti nationaliste.

Le statut de parti ultra-dominant de l'U.C. s'était ainsi mué insensiblement en abus de position dominante en surclassant nettement ses rivaux. En pratique d'ailleurs soulignent certains auteurs le parti ultra-dominant n'a toujours été qu'une étape sur la voie qui mène M. Prouzet, Le Cameroun, Paris, L.G.D.J., 1974, p. 57.

20 J. F. Bayart, L'Etat au Cameroun, Paris, P.F.N.S.P., 1979, p. 128.

21

Travaux du Congrès de Garoua tenu du 10 au 15 mars 1969, Les Presses, S.I.P.E., 1974, p. 114. 
irréversiblement au parti unifié ou unique. ${ }^{22}$ Dans ce cadre le Président Ahidjo s'est positionné en pédagogue suprême, expliquant la politique faite en son nom. Le risque, note Jean Lacouture, est que toutes les énergies politiques se dissipent dans une inépuisable scolarité adolescente ${ }^{23}$.

Ce qu'on peut dire est que le chemin de l'unité et de l'effritement de l'identité anglophone était pour le moins politiquement balisé. Et on ne pouvait plus dès lors redouter les effets de la partition fédéraliste. Il ne restait plus qu'à constitutionnaliser ces acquis. La révision constitutionnelle du 10 novembre 1969 en a constitué le prélude juridique.

\subsubsection{Le prélude constitutionnel de novembre 1969: L'usage politique du pouvoir constituant originaire}

Depuis la constitution du 4 mars 1960, le président avait largement fait une interprétation personnelle des lacunes et des ambiguïtés des révisions subséquentes. Ce qui engendra à divers moments des problèmes d'interprétation. Chaque fois que le chef de l'Etat donnait la sienne propre, elle était acquise, alors même que du strict point de vue juridique elle apparaissait parfois contestable. Par analogie on peut même dire qu'il avait repris mécaniquement les techniques de révision dont avait fait usage le général De Gaulle le 28 octobre $1968^{24}$.

Vraisemblablement, les articles 18 et 47 de la constitution du $1^{\text {er }}$ septembre 1961 apparaissaient comme les derniers obstacles à l'encadrement juridique des acquis politiques de 1966. Ils exigeaient en effet deux sous-majorités à savoir celles des groupes de députés élus dans chacun des deux États fédérés pour une loi portant révision constitutionnelle. La révision du 10 novembre 1969 institua la procédure référendaire en la matière. L'exposé des motifs qui mettait en avance l'impératif d'adapter la constitution "aux nécessités du Cameroun unifié" était en réalité une stratégie politique pour contourner les réticences des leaders anglophones. L'implication du pouvoir constituant originaire dans la procédure de révision devait exercer la "fonction manifeste" ${ }^{25}$ de légitimation du projet unitariste du président Ahidjo. Puisque désormais, c'était la nation qui, dans son ensemble et non plus la représentation des États fédérés au parlement, qui, sur l'initiative du chef de l'Etat, adopterait une éventuelle révision constitutionnelle ; le rejet du référendum par l'un des États

J. Lapalombara / M. Weiner, Political parties and political development, Princeton, 1966, pp. 301-302; lire aussi D. G. Lavroff, Les partis politiques en Afrique noire, Paris, P.U.F., 1972. Quatre hommes et leurs peuples, Seuil, 1969, p. 257.

24

De la même manière le Général Ch. de Gaulle avait initié la révision du 28 octobre 1962, esquivant le Parlement qui eut pu opposer une éventuelle irrecevabilité.

R. K. Merton, Elément de théorie et de méthode sociologiques, $2^{\mathrm{è}}$ Ed., Traduction française 1965 , pp. 126 et suivants. 
étant sans conséquence juridique puisque la majorité fédérale pouvait se dégager à partir de l'autre État pour l'ensemble des électeurs de la fédération. La loi $N^{\circ}$ 90/LF du 10 novembre 1969 avait institué une procédure de contournement plus qu'efficace. Par cette tactique machiavellienne, l'identité anglophone allait se noyer dans une sorte de pseudo-convivialité.

C'est sans doute l'intuition de voir juste et d'avoir raison dans les grands problèmes institutionnels du Cameroun indépendant qui a conduit le président Ahidjo à choisir la voie du référendum en court-circuitant le parlement et à forcer la solution sans trop se préoccuper de l'identité anglophone et des problèmes de procédure. Et c'est sans doute aussi parce qu'ils ont senti qu'ils n'avaient pas «ce génie de politique » que les leaders anglophones se sont rangés.

Sans verser dans un portrait quasi-narcissique de l'homme, il faut souligner le grand art oratoire du Président Ahidjo. Il connaissait l'art de la périphrase, des avertissements assurés, des incidences qui intriguent, des formules sibyllines qui flattent et font espérer et des expressions-choc qui portent.

Il va sans dire que la figure rhétorique du discours communautaire est essentiellement stratégique. On le ressentira d'ailleurs lors de présentation du projet de révision le 9 mai 1972 dans un discours à la fois ferme et chargé d'affectivité. Quelques extraits de son allocution à l'Assemblée Nationale en prélude au projet de constitution de 1972 traduisent à suffisance cette gymnastique d'esprit.

«(...) Je vois dans les rapports de collaboration harmonieuse qu'entretiennent le président de la République et l'Assemblée nationale, tous deux émanation de la souveraineté nationale, l'une des expressions originales de la démocratie camerounaise (...) » disait-il. ${ }^{26}$

En fait s'agissait-il d'une collaboration ? Celle-ci supposant un équilibre et un échange entre les organes constitutionnels. Or l'image adulatrice que s'était taillée le président Ahidjo s'était vite muée en acceptation d'où un type particulier de relations entre le chef de l'Etat et cette assemblée pusillanime. Pour reprendre la formule de Lasswell, on peut dire qu'il n'y pas eu échange qui suppose un double mouvement (poids et contrepoids) mais «allocation ${ }^{27}$. Lors de la conférence de Foumban, on a noté le ton presque suppliant dans les propositions du Premier Ministre anglophone Foncha, qui évoquait les suggestions $\mathrm{d}$ '"un jeune frère formulées à l'endroit de son grand frère" ${ }^{28}$. 
De même comme pour « inhumer » définitivement la fédération, le président le la République dresse un constat d'échec de cette structure. Il note en effet «la pesanteur que les structures fédérales exercent sur les efforts de développement du peuple camerounais (...) trois gouvernements et quatre assemblées entraînent inévitablement des dépenses importantes (...). Le budget du Cameroun Occidental qui connaît toujours le déficit ». Cet exposé de motifs traduisait sa "profonde conviction " du nécessaire dépassement des structures fédérales.

Ainsi la révision de novembre 1969 instituant désormais le recours au référendum pour toute révision constitutionnelle, ne dissimula qu'à peine un usage politique du constituant originaire.

Ces préludes juridico-politiques ne signifiaient rien d'autre que la marche inéluctable vers l'Etat unitaire dont le chant de cygne sera réalisé le 2 juin 1972. Les avis semblent partagés quant à la nature juridique de la révision constitutionnelle du 2 juin 1972 et à celle du 18 janvier $1996^{29}$. Notre profonde conviction est que dans cette phase de l'histoire politique du Cameroun, la production normative a été largement caractérisée par l'institutionnalisation de la fraude constitutionnelle. C'est du moins dans une certaine mesure l'avis du Professeur Kamto ${ }^{30}$ par opposition à l'opinion des "Juristes organiques" restés de marbre face à ces critiques pourtant pertinentes. De manière surabondante, on peut même affirmer que la rupture du contrat politique était consommée entre la communauté anglophone et la communauté Francophone.

\subsection{Les constitutions du 2 juin 1972 et du 18 janvier 1996: La rupture du contrat politique ou l'initiation à la pratique de la "fraude constitutionelle"}

On peut situer le début de la pratique frauduleuse en matière de révision constitutionnelle à partir du texte du $1^{\text {er }}$ septembre 1961. En effet, la constitution du 4 mars 1960 qui instituait un régime de type parlementaire était peine mise en place qu'elle fut remplacée par celle du $1^{\text {er }}$ septembre 1961, instituant un régime complexe à base du fédéralisme classique. A l'évidence, la nature du régime avait fondamentalement changé. Il va sans dire que le texte de 1961 devrait recevoir la dénomination de "nouvelle constitution" assortie d'une appellation convenable.

Elle se désigne paradoxalement mais techniquement "Loi nº 01-24 du $1^{\text {er }}$ septembre 1961 portant révision constitutionnelle et tendant à adapter la constitution actuelle aux nécessités

F. Mbombe, Constitution du 2 juin 1972 révisée ou nouvelle constitution ?, A.A.S.P., F. Ebert, Yaoundé, 1997, pp. 16 - 53.

30

Lex Lata, N023 -024, Février - Mars 1996, p. 17 et suivantes. 
du Cameroun unifié". On pouvait s'attendre à une dénomination plus conforme aux profondes mutations qu'a connues le Cameroun en 1961 puisque ce texte établissait une constitution bien nouvelle. On ne peut hésiter ici de parler de fraude à la constitution.

Depuis lors, on assistera de manière incessante à une sorte d'institutionnalisation de cette pratique frauduleuse. Sans doute correspondait-elle aux projets politiques des dirigeants dont l'obsession pour l'unité nationale inclinait à des pratiques peu orthodoxes.

En tout état de cause, les constitutions du 2 juin 1972 et du 18 janvier 1996 ont consacré une interprétation personnelle des ambiguïtés de la constitution et une inhumation définitive du fédéralisme par un usage politique du droit.

\subsubsection{La constitution du 2 juin 1972 et la prédominance des préoccupations unitaristes: Les usages politiques du droit ou les difficultés d'une interprétation}

Les points de vue sont du reste partagés sur la nature de cette constitution qui vient de manière surabondante modifier la forme de l'Etat en forçant une unité qui allait trancher définitivement avec le "contrat politique" de septembre 1961. Des voix autorisées s'élevèrent pour critiquer vivement ce « hold-up » constitutionnel ${ }^{31}$.

«Il semble même, souligne le Professeur Kamto, qu'Ahmadou Ahidjo n'ait jamais conçu le fédéralisme autrement que comme un fédéralisme de transition $»^{32}$. En dénonçant le fédéralisme comme «un handicap au développement rapide du pays » ${ }^{33}$, le chef de l'Etat ne faisait plus mystère des orientations qu'il entendait imprimer à l'acte constitutionnel de 1972, mais en a tenu secret le projet. ${ }^{34}$ On peut dire à la suite de Louis Favoreu que le constitutionnalisme camerounais semble manifestement saisi par la politique; car de l'illusion de la prise en compte du biculturalisme, on en est arrivé par touches successives à une "assimilation pure et simple" de la communauté anglophone. En réalité, il faudrait y voir une part de «personnification de l'expression politique ${ }^{35}$ par les initiateurs de ce texte qui pousse à son terme confine l'autoritarisme.

C'est le cas de John Ngu Foncha, qui démissionna en 1970 pour marquer sa désapprobation face à cette façon de procéder.

M. Kamto, Dynamique constitutionnelle du Cameroun indépendant..., Revue juridique africaine, P.U.S., 1995, p. 11.

Discours de présentation du projet de constitution cité.

E. Mbarga, Evolution politique du Cameroun en 1972: référendum du 20 mai 1972 et la formation de la République Unie du Cameroun, Annales de la Faculté des Droits, UFC, 1973, p. 19 et suivantes. 


\subsubsection{L'interprétation personnelle des ambiguïtés de la constitution}

L'obsession unitaire qu'on colle à l'oeuvre du président Ahidjo apparaît surtout dans les œuvres hagiographiques. Car après avoir gommé toutes les aspérités sécessionnistes, il se posait désormais en «pédagogue suprême». La culture politique «de participation» (1961-1966) se muait ainsi insensiblement en «culture de sujétion » ${ }^{36}$. On pouvait redouter dès lors une propension personnalisante du pouvoir. Ces appréhensions semblaient d'autant plus fondées que le chef de l'Etat avait fait du texte de 1972 une interprétation bien personnelle, qui avait inévitablement abouti à l' "absolutisme présidentialiste" ${ }^{37}$.

Au fond les ambiguïtés achoppaient sur la nature du texte de 1972. Ayant tenu longtemps secret son projet, les partenaires anglophones ne pouvaient en imaginer les contours. Se fondant pour l'essentiel sur l'article 47 de la constitution fédérale de 1961, les personnalités politiques anglophones décrièrent véhémentement le procédure peu orthodoxe utilisée par le Chef de l'Etat. Car cet article précisait in fine que la révision devait « être votée à la majorité simple des membres composant l'Assemblée Fédérale à condition toutefois que cette majorité comporte des représentants à l'Assemblée Fédérale de chacun des États fédérés. » En esquivant cette disposition par ailleurs rédigée en termes péremptoires, s'agissait-il d'une fraude à la constitution, d'un détournement ou d'un contournement de procédure ? Ces expressions ont chacune un sens et ne sont pas par conséquent interchangeables.

L'argument de la fraude eut pu prospérer si le président Ahidjo n'avait pas fait table rase de la constitution fédérale de 1961. Ici l'hypothèse d'une fraude à la constitution n'est soutenable que si la notion elle-même est entendue lato sensu "comme visant toute opération de renouvellement partiel ou total d'une constitution" ${ }^{38}$. Il s'agit évidemment pour le professeur Kamto d'une nouvelle constitution puisqu'elle ne s'est pas fondée sur la constitution précédente. Au demeurant, la pertinence d'une telle thèse reste à expliquer. En effet on peut considérer que la Reforme de novembre 1969 qui instituait le recours au référendum et qui fondait par ailleurs la procédure utilisée par le Chef de l'Etat était une révision de la loi fondamentale de 1961. Dans l'esprit du constituant l'avis des premiers ministres des États fédérés et des Députés de l'Assemblée fédérale revêtait désormais le caractère d'une suggestion purement consultative. Le constituant de 1972 s'est donc référé à la constitution de 1961 de manière sélective, en usant uniquement de la reforme de novembre 1969. Cette argumentation entrainait les juristes anglophones sur le terrain inconnu du droit constitutionnel français qui contraste fort avec une formation de Common law.

A. Gabriel/B. Powell, Comparative politics, developmental approach, Princeton, 1966, p. 146.

K. A. Kontchou, Données de base, évolution constitutionnelle, Constitutions africaines, Vol. 1, Sous la direction de F. REYJENS, Bruxelles, Bruyland, p. 3.

M. Kamto, op.cit. (note 32), p. 15. 
Qui plus est, les personnalités anglophones ont situé le problème sur le terrain de conflit des lois entre une disposition spéciale et une disposition générale (révision du 10 Novembre 1969 et l'article 47 de la constitution du $1^{\text {er }}$ septembre 1961) pour en conclure à la prévalence de la première au nom du principe "spéciali generalitus derogat". Si même on retenait cette argumentation, laquelle des deux dispositions allait revêtir la forme spéciale ? Puisque la reforme de 1969 laissait apparemment le Chef de l'Etat seul juge de l'opportunité de l'intervention. Incontestablement, cet argumentaire nous perd en conjecture. Il ne tient d'ailleurs qu'à un fil: celui du Droit saisi par la politique.

En tout état de cause, il s'est agi en 1972 de l'établissement d'une nouvelle constitution. La forme de l'Etat avait fondamentalement changé et le recours au Référendum l'en avait attesté. L'issue de cette consultation semblait bien connue, puisque ce référendum assimilateur impliquait désormais un décompte séparé des suffrages valablement exprimés indifféremment de l'appartenance à l'un ou l'autre État fédéré.

Le débat sur l'utilisation d'une telle procédure engagée sous une autre forme dans le contexte français ${ }^{39}$ quant à l'opportunité du recours à l'article 11, a suscité la même controverse. La thèse de l'inconstitutionnalité s'est avérée dominante dans le cas d'espèce ; seul Pierre Lampué devait exprimer une opinion différente ${ }^{40}$. On peut dire que le précédent du 2 Juin 1972 a constitué une "coutume inconstitutionnelle". Après avoir définitivement arrondi les angles de la constitution unitaire, le Président Ahidjo s'attellera à museler tous les nostalgiques du fédéralisme.

\subsubsection{La manipulation constitutionnelle et la suspicion conviviale}

Le paternalisme pédagogique du Président Ahidjo et son intuition de voir toujours juste avaient très tôt occasionné une défection au sommet de l'Etat. La démission solitaire du Vice-président John Ngu Foncha en 1970 traduisait du reste dans une mesure significative l'ampleur de la suspicion conviviale entre les communautés anglophone et francophone. Cette démission assimilable à l'attitude de Gribouille ${ }^{41}$ a permis d'effacer les dernières velléités fédéralistes et a ouvert droit à une nouvelle recomposition du puzzle politique.

La révision constitutionnelle de 1975 qui rétablissait le poste de Premier Ministre, successeur constitutionnel (à partir de Juin 1979) instaurait une "tryarchie" ${ }^{42}$ politiquement

P. Lampué, Le mode d'élection du Président de la République et la procédure de l'Article 11, R.D.P., 1962, p.931.

41 Personnage mythologique, symbole de la maladresse brouillonne.

42

La "tryarchie" n'est pas entendue ici dans le sens d'un triumvir, mais dans le sens d'un positionnement géopolitique à la tête de l'Etat. 
connotée. La nomination de M. Biya à ce poste revêt une double signification stratégique: la volonté de déconcentrer le pouvoir présidentiel sans toutefois créer un véritable bicéphalisme de l'exécutif. Plus déterminante est cette stratégie d'évitement des personnalités anglophones sur qui continuait de peser la suspicion séparatiste. Ne pouvait-on pas s'attendre pour des raisons d'équilibre à la désignation d'un anglophone compte tenu du biculturalisme hérité de l'administration coloniale. La nomination de M. Biya désormais successeur constitutionnel traduisait une sorte de "francophonisation" du pouvoir assortie d'une alternance géopolitique entre le Nord et le Sud au détriment des personnalités anglophones réduite à une présidence figurative au sommet de l'Etat. Cette observation est d'autant plus vraisemblable qu'en 1982 le Président Biya nomma au poste de Premier Ministre, M. Bello Bouba originaire du grand Nord. N'eut été le complot du 22 Août 1982 qui avait profondément terni l'image de l'ancien Président de la République, le Président Biya aurait indubitablement consolidé cette alternance géopolitique en conservant la disposition relative au dauphin constitutionnel ${ }^{43}$. Puisqu'en supprimant cette disposition, $M$. Biya "avait retiré l'échelle derrière lui une fois arrivé au sommet" ${ }^{44}$. La psychose du coup d'Etat était pour le moins fondée et les événements du 6 Avril 1984 venaient traduire à la fois la volonté d'une maîtrise totale du pouvoir par le nouveau Président et les inquiétudes de "son illustre prédécesseur" face aux velléités "iconoclastes" de son successeur.

C'était le temps des incertitudes. Et le Chef de l'Etat trouva une parade qui a suscité bien des appréhensions. Pour la première fois un anglophone était appelé selon les termes de l'article 7, à achever le mandat présidentiel en cas de vacance du pouvoir. Était-ce un point d'honneur pour le pouvoir en quête de légitimité ? Une telle hypothèse semble un peu tirée sur la comète . D'abord le Chef de l'Etat se serait assuré du loyalisme de Salomon Tandeng Muna alors Président de l'Assemblée nationale et l'aménagement constitutionnel le confortait dans sa position de deuxième personnalité de l'Etat et de "successeur constitutionnel temporaire". ${ }^{45}$ Cette incertitude a été très vite dissipée avec l'élection de Monsieur Cavaye Yegue Djibril à la tête de l'Assemblée nationale, après que le chef de l'Etat eût précipité la nomination d'un premier ministre anglophone (désormais sans héritage constitutionnel). Ce jeu de "cartes biseautées", par delà les faux-semblants de l'équilibre, participe de la "francophonisation" du pouvoir et de la suspicion conviviale entre les deux communautés. La désignation d'un premier ministre anglophone dans une structure où le Président de la République monopolise toute la fonction exécutive argumente davantage ce point de vue. ${ }^{46}$

Voir Article 7 de la loi portant révision constitutionnelle du 4 Février 1984.

M. Kamto, op.cit. (note 32), p. 28.

Article 7 de la constitution du 2 Juin 1972.

V. Miaffo, Le Président de la République et les constitutions du Cameroun », AASP/GRAP Fondation Friedrich Ebert 1996, pp. 252 - 281 
La distribution du pouvoir au sommet traduit parfaitement cette reproduction atavique du système à l'œuvre depuis l'indépendance. Qu'on nous concède ce néologisme - peut-être rébarbatif, mais significatif - "d'anglophobie" dans la configuration politique au sommet de l'Etat. A ce niveau d'analyse se révèle l'aspect brouillon des reformes constitutionnelles depuis l'indépendance. ${ }^{47}$ Cette méfiance incline à conclure que la démocratisation dont parlent aujourd'hui les gouvernants avec un accent tonique n'aura été qu'une arlésienne ; la possibilité pour une personnalité anglophone d'accéder à la magistrature suprême revêtant le caractère d'une faible probabilité.

Une telle suspicion servira de prétexte à toutes les réticences lors de l'élaboration de la constitution du 18 Janvier 1996. Non seulement l'idée du fédéralisme soutenue par les anglophones a été d'emblée évacuée et la procédure mal ficelée, mais de nouveaux concepts aux contours au demeurant flous sont apparus, donnant ainsi à réfléchir sur les véritables intentions de leurs initiateurs. Le clivage classique anglophones/francophones se double aujourd'hui de nouvelles factures sociales (minorités, autochtones, allogènes) dont la constitution du 18 janvier 1996 s'est attelée à en assurer l'encadrement juridique.

\section{La reforme constitutionnelle du 18 janvier 1996: L'étouffement du séparatisme et l'encadrement juridique de nouveaux clivages sociaux (minorité, allogène, autochtone...)}

La mise en place des nouvelles institutions a recelé d'énormes contradictions, des déclarations et des contre-déclarations qui embrouillent le juriste pour qui les expressions peuvent se rapprocher sans pour autant être interchangeables. Les clauses de la tripartite par rapport à la suite des événements étaient pour le moins lapidaires. Cette rencontre qui réunissait à la fois le parti au pouvoir, les partis d'opposition, les représentants de l'administration, les représentants de la société civile avaient élaboré un échéancier qui avait fait l'unanimité des participants: un comité technique avait été mis en place pour élaborer un avant-projet de constitution à soumettre à la conférence pour discussion et approbation, lequel projet devait être soumis en dernière instance au référendum. Un tel schéma apparaissait comme une solution de rechange à la conférence nationale et pour laquelle le gouvernement avait opposé un refus catégorique. ${ }^{48}$ Ce calendrier plus réaliste et plus commode à l'intensité des revendication sociales et qui eut pu aboutir à un texte consensuel n'avait hélas duré que le temps d'une décrispation de l'atmosphère politique rendue délétère par l'élection présidentielle anticipée du 11 Octobre 1992. Le lancement tardif de ce débat le 23 Mars 1993 devait

L. Sindjoun, Cameroun: le système politique face aux enjeux de la transition démocratique (19901993), l'Afrique politique, Karthala, 1994, pp. 143 - 161.

48

Le chef de l'Etat se trouva apparemment pas d'objet à la C.N.S assimilée à « un coup d'Etat civil » 
à la longue s'inscrire dans un univers discursif sémantiquement controversé. ${ }^{49}$ Le "grand débat" annoncé allègrement par le porte-parole du gouvernement s'est transformé subrepticement - par une étrange gymnastique verbale - en "large débat". Apparemment le chef de l'Etat s'était rétracté et avait lâché son Ministre d'Etat; car en réalité l'expression "grand débat" par sa tonalité joignait une conception abrupte voisine d'une certaine imprudence ${ }^{50}$. On eut pu l'assimiler à la redoutable Conférence nationale souveraine. Cette attitude allait exercer un tropisme sur la suite de débat qui s'est fait pour l'essentiel par téléphone et par fax. Le texte qui en est résulté était manifestement bien loin de donner satisfaction tant sur la forme que sur le fond.

En évacuant les problèmes de procédure, qui au demeurant, s'est avérée mal ficelée, la constitution du 18 janvier 1996 insinue une énorme contradiction. En même temps qu'elle affirme l'indivisibilité de l'Etat et "l'intangibilité" de son caractère unitaire, elle assure de manière feinte la promotion des nouveaux clivages sociaux. Cet exercice de funambule est parfois révélateur de l'impuissance de l'Etat à contenir la progression des référants socioculturels. Sans décomposer la nature unitaire de l'Etat, le constituant du 18 janvier 1996 a modulé une échancrure vers l'inscription des différences dans la droit positif. La phobie du séparatisme reste donc entière.

\subsection{L'étouffement du séparatisme}

Le problème de la forme de l'Etat a été remis en surface lors du débat sur la constitution du 18 Janvier 1996. Pour l'essentiel, deux thèses radicalement opposées animaient la controverse: le gouvernement et certains partis de l'opposition farouchement opposés au fédéralisme ne concevaient le réaménagement de la forme de l'Etat que dans le sens d'une décentralisation poussée, voire d'une régionalisation. Si l'unanimité s'est faite au sein de la communauté anglophone sur un retour au fédéralisme, elle restait au demeurant partagée sur le nombre d'États fédérés. ${ }^{51}$ Leur argumentaire reposait à la fois sur ce qui est convenu d'appeler - sans que la formule ait seulement le brillant du paradoxe - le "troc dolosif" de 1972 et sur les effets pervers du système Jacobin qu'ils ont jugé préjudiciable à leur identité.

La déclaration du Ministre d'Etat chargé de la communication annonçant la tenue d'un "grand débat" avait à peine suscité l'espoir d'une discussion ouverte, que le chef de l'Etat jetait un pavé dans la marre en précisant qu'il s'agirait d'un "Large débat" dont le bilan prouve aujourd'hui son caractère clos.

F. X. Mbome, Processus d'élaboration de la constitution du 18 Janvier 1996, Lex Lata, Vol. 030, 1996, pp.3 - 8.

L'opposition est bien apparent entre le A.A.C. qui soutient un fédéralisme à 2 et les chefs traditionnels du Sud-ouest qui prônent un fédéralisme à dix. 
Au moment où la sérénité s'installait déjà dans la perspective d'un débat ouvert après les concessions parfois compromettantes, le chef de l'Etat fera un pavé dans la mare en précisant que « l'Etat unitaire est la forme d'Etat qui convient. Le gouvernement avait toutes les raisons de redouter un retour au fédéralisme en raison de la vigueur de ces revendications. Et, la création de l'Etat "d'ambazonie" par la branche radicale du Southern Cameroon National Council (en réaction au refus du gouvernement) donnait plus de corps à cette supposition. Le pouvoir a cru devoir répondre à ces incessantes revendications en optant pour une solution médiane: la forme régionale qui manifestement présente une complexité qui cache mal l'intention de maintenir une tutelle pesante sur les régions, révélait l'intangibilité du caractère unitaire de l'Etat. Le bicéphalisme entretenu volontiers au niveau de la région entre le président du conseil et le gouverneur (une sorte de délégué du pouvoir central) le démontre à suffisance. ${ }^{52}$

L'étouffement du séparatisme a également opéré par une stratégie de positionnement des leaders anglophones "Organiques". La nomination de Simon Achidi Achu à l'issue de l'élection législative de 1992 et modérateur du débat était pour le moins symbolique. Certains chefs traditionnels ${ }^{53}$ anglophones étaient mis à contribution pour conforter l'idée que le fédéralisme n'était qu'un tremplin pour le projet séparatiste de ses tenants. De même, la démission de trois juristes anglophones du comité technique mis en place par le chef de l'Etat le 17 Mai 1993 n'avait apparemment pas d'incidence sur les 6 restants ${ }^{54}$ dont les prises de position était bien connues. On peut même supposer que le fédéralisme à 10 États proposé par les chefs traditionnels du Sud-ouest entrait dans cette stratégie de diversion pour esquiver le grand clivage bipolaire qu'insinuaient les propositions du "All Anglophone Conference" ; sinon comment expliquer la relative indulgence du pouvoir face à cette suggestion qu'il avait tôt fait d'évacuer de l'ordre du jour. Le Chef de l'Etat avait fait bon usage de la technique du "passing shoot" en attirant son adversaire pour mieux le lober. A l'évidence, le fédéralisme est apparu pour le pouvoir comme une "rose pleine d'épines".

Qui plus est dans une logique empreinte de diversion au sujet de l'instauration du fédéralisme, les "entrepreneurs politiques" ont créé une fracture ouverte au sein de la communauté anglophone, dont le clivage ethnologique a contribué à agrandir le point d'impact ${ }^{55}$. Ici l'ethnie prend toute sa connotation dévalorisante. En effet après avoir créé artificiellement le concept "Anglo-Bami" ${ }^{56}$ pour désigner un ennemi fictif dans un contexte où le pays

52

53

54

55

56

Article 57 al. 4 de la constitution du 18 Janvier 1996.

On peut citer le Fon Angwafoir; le Fon de Balikoumbat

F. Mbome, Constitution du 2 Juin 1972 révisée ou nouvelle constitution, La Réforme constitutionnelle du 18 Janvier 1996: aspects juridiques et Politiques, AAPS, F. Ebert 1996 p. 21.

Les populations anglophones du Nord-ouest (appartenant au grand groupe grassfields) contrastent fort avec celle du Sud-ouest plus proches des Sawa.

Le concept a fait du chemin depuis l'instauration du multipartisme. 
a failli basculer dans la guerre civile, les stratèges ont poussé la logique à son terme en opposant les population Sawa du Sud-ouest aux populations Grassfields dont fait partie la province anglophone du Nord-Ouest. Du coup, le problème anglophone s'est trouvé dilué dans ces lignes ethnologiques tracées au cordeau.

La nomination d'un premier ministre anglophone d'origine Sawa à l'issue des élections municipales d'Octobre 1996 revêt une double signification: d'abord elle consacre la rupture d'une "coutume politique" à l'œuvre depuis l'indépendance. Les personnalités anglophones du Nord-ouest avaient en effet ravi la vedette à celles du Sud-ouest à l'époque manifestement opposées au projet unificateur du Président Ahidjo. On se souvient que Monsieur Foncha était apparu comme le seul interlocuteur valable de la communauté anglophone en raison de son adhésion à ce projet. Une adhésion qui s'était très vite muée en résignation ${ }^{57}$. Ensuite cette nomination s'est révélée comme une rétribution électorale au détriment d'une région hostile très tôt acquise à la cause de l'opposition. Le but inavoué étant sans doute d'opérer une fracture au sein de cette communauté pour en offrir l'image d'un bloc divisé sur la perception des problèmes politiques nationaux. Le Premier Ministre, Monsieur Peter Mafany Musonge en convoquant les chefs Sawa à des intervalles réguliers à l'approche de l'élection législative du 17 Mai 1997, avait la profonde conviction que cette manœuvre agrandirait davantage la fracture entre le Nord-ouest et le Sud-ouest.

La large victoire du parti au pouvoir (R.D.P.C.), au demeurant contestée par l'ensemble de l'opposition, prouve à tous les moins que les connecteurs ensemblistes de la communauté anglophone ont lâché. Et alors on a assisté à une "dilapidation progressive" de son identité $^{58}$. Il est intéressant de noter que la quête de l'électorat francophone par le S.D.F. a indirectement joué sur la vigueur des revendications identitaires. Son caractère "attrapetout" ${ }^{59}$ a gommé insidieusement l'élan "révolutionnaire" et l'a transformé progressivement en "parti tribunicien"60. De surcroît, les contradictions internes ne l'ont pas toujours épargné: la participation parlementaire du S.D.F. après sa "piètre" performance à l'élection législative du 17 Mai 1997 a suscité (au regard de la non participation auparavant prônée par son leader) une vive controverse au sein de cette formation politique. Politiquement, il eut été mal indiqué de boycotter le Parlement, tirant argument du fait que, les élus du S.D.F. s'opposaient à la fois en fonction de leur division culturelle et linguistique et de leurs motivations, de leur stratégie et de leur appartenance ethnique. Il n'empêche qu'au

Il démissionna du poste de Vice-président de la République en 1970 en réaction à la politique unificatrice du Président Ahidjo. (ed), Political development, Princeton, 1966, pp. 171 - 200.

G. Lawau, A la recherche d'un cadre théorique pour l'étude du Parti Communiste Français, R.F.S.P., 1968, p. 26. 
delà des entrecroisements des tendances, les anglophones conservent encore une forte conscience de leur spécificité par rapport aux élus francophones. Aussi a-t-on trouvé juste cette participation qui eut été fatale dans l'hypothèse suicidaire d'un boycott. Le pouvoir en a évidemment tiré les dividendes politiques; puisque les 43 Députés du S.D.F. ne modifient en rien la configuration politique d'un Parlement où il s'est adjugé une majorité absolue. Toutefois, malgré l'ultra-dominance du parti au pouvoir, ce Parlement offre désormais l'image d'une Assemblée multipartisane qui dilue l'identité anglophone dans un puzzle politique autrement complexe.

On passe ainsi (comme au Canada) insensiblement du bilinguisme au multiculturalisme ${ }^{61}$, d'une identité camerounaise bipolaire à une identité multipolaire sous-tendue par la balkanisation ethnique. Dans ce contexte, la minorité anglophone ne sera plus perçue comme l'éternel trouble-fête, comme la fausse note dans le concert politique camerounais. Désormais, le pouvoir central devra manœuvrer le kaléidoscope que constitue la nouvelle configuration politique. Aussi le pouvoir s'est-il attelé à constitutionnaliser ces nouvelles formes de clivages sociaux en reposant son argumentaire sur la protection des minorités et des autochtones.

\subsection{La constitutionnalisation de nouvelles formes de clivages sociaux (minorités, allogènes, autochtones...): l'imprécision des notions et le risque de dévoiement conceptuel}

La densité de la Constitution du 18 Janvier 1996 montre que le constituant camerounais n'a voulu laissé en friche aucun aspect de la vie politique nationale. C'est d'ailleurs "la tendance générale aujourd'hui en particulier dans les pays sous développés d'élaborer des textes constitutionnels détaillés, et les plus précis possibles afin d'éviter les flottements et arbitraires dus aux lacunes et les incertitudes liées à l'interprétation d'un texte général ou trop vague", nous fait remarquer le Professeur Maurice Kamto ${ }^{62}$.

Le concept de minorité jusque là réservé à la communauté anglophone et qui justifiait par ailleurs toutes les réticences, a par duplication enfanté, selon une opinion largement diffusée, une conception "plurielle de la citoyenneté" ${ }^{63}$ : désormais il existerait des camerounais autochtones, allogènes ou minoritaires. Nous nous abstiendrons de psychanalyser les

H. D. Forbes, Canada: from bilingualism to multiculturalism, Journal of democracy Vol. $4, \mathrm{~N}^{\circ} 4$. Octobre 1993, pp. 69 - 84.

62

Dynamique constitutionnelle ..., op.cit., p. 39.

63

S. L. Donfack, Existe-t-il une identité démocratique camerounaise ? La spécificité camerounaise à l'épreuve de l'universalité des droits fondamentaux », Revue camerounaise de Science politique, Vol. 1, Fév. 1996, p. 39. 
pulsions qui motivent une telle perception pour placer les choses dans leurs justes proportions.

Dès son préambule, la Constitution du 18 Janvier 1996 déclare dans une formule imprécise que: "l'Etat assure la protection des minorités et préserve les droits des population autochtones conformément à la loi". Il faudrait souligner au-delà des controverses qu'a suscité l'introduction de ces concepts dans la loi fondamentale qu'ils ne sont pas une spécificité camerounaise. D'ailleurs ne participe-t-il pas aujourd'hui de la construction d'une "démocratie consociative" dans les nations occidentales. Leur reconnaissance n'est donc pas une onction constitutionnelle pour une idée malsaine. En effet, la protection des minorités et devenue à la fois un élément de la démocratie et un facteur de la paix et de la stabilité des Nations bien que depuis la Seconde Guerre Mondiale jusqu'à la fin des années 80 cette question ait été mise entre parenthèses ${ }^{64}$. La crainte de plusieurs États face à ce qu'ils considéraient comme un danger pour leur souveraineté et leur intégrité territoriale justifie le peu d'intérêt jusque-là accordé à cette notion. Or une telle attitude peut conduire malheureusement à ce qu'on veut éviter. Le génocide Rwandais et Burundais, le drame de la Yougoslavie, les velléités sécessionnistes aux Comores, en constituent le témoignage le plus éclatant.

Plusieurs instruments juridiques internationaux ont tenté désespérément de cerner avec précision cette notion. ${ }^{65}$ On peut tout de même regretter le fait que ces textes procèdent par énumération. L'article $27 \mathrm{du}$ Pacte des droits civils et politiques semble accentuer cette imprécision en renvoyant indirectement la définition de ce concept au droit interne: "dans les États où il existe des minorités ethniques, religieuses ou linguistiques, les personnes appartenant à ces minorités ne peuvent être privées du droit d'avoir en commun avec les autres membres de leur groupe, leur propre vie culturelle, de professer et de pratiquer leur propre religion, ou d'employer leur propre langue".

C'est sans doute ce manque de précision qui alimente les appréhensions quant à sa réception par le droit constitutionnel camerounais par delà les débats passionnés qui situent la notion dans le champ de l'ethnicité. Ce malentendu s'est fait ressentir au niveau du Comité

A. L. Sicilianos, Les Minorités: minorités nationales, populations autochtones, dans: Les Droits fondamentaux, AUPELF / UREF, Université de Nantes - Université de Paris Nanterre, 1994, p. 28. Voir aussi A. Lipjphart, Consociational democracy, World Politics XXI (2) 1969, pp. 207 213.

On peut citer entre autres le Pacte des droits civils et politiques de 1966, l'Article 5 de la Convention de l'UNESCO pour la lutte contre les discriminations dans l'enseignement, l'Article 30 de la Convention relative aux Droits de l'enfant, la Déclaration des droits des personnes appartenant à des minorités nationales ou ethniques, religieuses et linguistiques. 
des droits de l'homme des Nations Unies ${ }^{66}$. Dans certaines communications présentées au Comité en application du protocole facultatif consacré à l'article 27 , le problème des minorités a été confondu avec le droit des peuples à disposer d'eux-mêmes, énoncé à l'article $1^{\text {er }}$ du Pacte. En outre, dans les rapports présentés par les Etats-parties conformément à l'article 40 du Pacte, les obligations imposées aux États parties par l'article 27 ont été parfois confondues avec les devoirs qu'ils ont en application du paragraphe 1 et de l'article 2 de garantir les droits reconnus dans le Pacte sans distinction aucune, ainsi que le Droit à l'égalité devant la loi et à une égale protection de la loi énoncée à l'article 26.

Au fond, la jouissance des droits énoncés à l'article 27 ne porte pas atteinte à la souveraineté et à l'intégrité de l'Etat-partie. Toutefois, l'un ou l'autre des droits consacrés dans cet article - par exemple - la droits d'avoir sa propre vie culturelle, peut consister en un mode de vie étroitement lié au territoire et à l'utilisation de ses ressources. Cela peut être vrai en particulier des membres des communautés autochtones constituant une minorité. Dans le contexte camerounais, l'introduction de ces notions dans l'ordonnancement constitutionnel a révélé toute la vigueur des clivages ethniques. ${ }^{67}$ En réalité, le problème des minorités, des allogènes et des autochtones est né de l'extrême mobilité de certains groupes ethniques vers les villes cosmopolites du Cameroun. Les Bamiléké qui sont les plus enclins à des tels mouvements, ont dû par leur "dynamisme" et leur "mentalité acquisitive"68 provoquer les frustrations des populations autochtones. Il s'agit, ce qui est la triste évidence d'un problème foncier. Une étude topographique des implantations des populations dans les provinces du Littoral, du Sud, du Centre et du Sud-ouest indique que les groupes ethniques qui se réclament avec un enthousiasme tonique du droit des minorités sont ceux qui ont été territorialement supplantés par les populations dites "allogènes" ${ }^{69}$. L'on se souvient qu'à l'avènement du multipartisme la lutte politique s'était cristallisée autour de "la récupération des terres des ancêtres dolosivement cédées aux allogènes". Ce sont ces mêmes prétentions qui sont reprises de façon biaisée par les hommes politiques. ${ }^{70}$

La réception de la notion de minorité dans le droit constitutionnel camerounais semble alors relever d'un mimétisme circonstanciel. ${ }^{71}$ D'abord le constituant semble lier

Observations générales 23 (Article 27) du Comité des droits de l'homme; 50e session 1994, H.R.I./G.E.N./1/R.E.V.1, 29 Juillet 1994.

C'est du moins ce que leur reprochent les populations autochtones.

A ce sujet on peut citer les aires culturelles Sawa, Béti et Boulou.

70

Lors de la marche anti Bamiléké en 1996, Me Douala Moutome, ci-devant Ministre de la Justice et originaire du Littoral déclarait sans œillères que cette marche traduisait l'attitude "d'un charbonnier désireux d'être maître chez lui". 
l'émergence du problème des minorités ethniques à la démocratisation, comme pour célébrer le système monopartisan dans lequel, fait-il croire, la minorité était inexistante. Puisque dans ce contexte, les dirigeants africains dans des discours mobilisateurs faisaient "gober" un unanimisme illusoire. Ensuite, les "non-dits" de la disposition constitutionnelle y afférente montre que le pouvoir entend donner un sens spécifique aux notions de minorité et d'autochtone; ce qui incline à réfléchir sur les véritables intentions du constituant. Enfin, rédigée sous la forme d'une périphrase en renvoyant à une loi ultérieure la précision de ces concepts, ladite disposition laisse malheureusement libre cours à des interprétations subjectives propres à en dévoyer le sens. Sans doute le pouvoir central profondément atteint par les revendications sociales a-t-il voulu s'attirer quelque sympathie en s'improvisant défenseur des droits des minorités.

Or, sous d'autres cieux, la jurisprudence aussi bien que la doctrine classent le droit des minorités et des autochtones dans la rubrique "des inégalités compensatrices" ${ }^{72}$. Il s'agit aux USA de l'"Affirmative action"; les tribunaux ont créé des conditions favorables et incitatives à l'adoption des plans d'"Affirmative action" par les entreprises privées. Cette action a été sanctionnée en 1979 dans une importante décision de la Cour suprême: "United steelworkers of America versus Weber". Dans cette décision, la Cour suprême débouta un employé blanc, qui accusait son employeur de l'avoir discriminé en instaurant un plan d'"Affirmative action" qui offrait à des employés noirs, la possibilité de participer à un programme de formation dont il avait été écarté. Si les dichotomies (minoritaire-majoritaire et autochone-allogène) sont appréhendées dans le sens d'une absolue fixation ethno-territoriale des citoyens, il y a fort à parier qu'on débouchera sur une discrimination négative. De même la thèse de "l'inégalité compensatrice" dont on use généralement comme exposé de motifs ne correspond pas toujours à la doctrine qui les considère comme "un remède aux discriminations dont les groupes ont souffert dans le passé ... "

Pour que les inégalités compensatrices soient admises comme telles, elles doivent être accessoires aux droits essentiels à l'ordre démocratique ; ensuite le critère distinctif tel que la race, le sexe et l'ethnie doit être pertinent et avoir un rapport de causalité avec l'objectif légitime poursuivi. Il faut enfin qu'elles ne soient pas fondées sur une présomption d'infériorité raciale ou ethnique. Ces distinctions qui visent par ailleurs "à parer aux futures discriminations" ${ }^{73}$ révèle que le contexte camerounais, si ce concept n'est pas manipulé avec beaucoup de dextérité, il servira d'alibi confortable à la défense des intérêts inavoués.

P. Schutter, Egalité et différence: le débat constitutionnel sur la discrimination positive aux Etats Unis, R.T.D.H., 1991, p. 336. Voir également P. Noblet, L'Amérique des minorités, les politiques d'intégration, Paris, Harmattan, 1993, et N. Glazer, Affirmative action, discrimination, ethnic inequality and public policy, Harvard, New edition, 1987.

V. Ergec, Les inégalités compensatrices, dans: L'effectivité des droits fondamentaux dans les pays de la communauté francophone, Paris, 1991, pp. 87 - 93. 
Sur cette base, il sera difficile non seulement de promouvoir les droits des minorités et des autochtones, mais aussi d'en consacrer la pleine justiciabilité. Aussi convient-il d'interpeller les tenants de la constitution du 18 Janvier 1996 sur les appréhensions des uns et la déterminations des autres sur cette épineuse question des minorités: de même qu'on ne devrait en prendre peur en recherchant d'illusoires subterfuges, comme si on pouvait se soustraire d'une réalité qui s'impose à tous, de même toute discrimination est une amputation faite au corps de la Nation.

En tout état de cause, le chemin de la reforme est désormais tracé. Il faut du temps. Et, le temps ne pardonnant rien à ce qui est fait sans lui, "ce n'est pas une sorte de sommation brutale" que la notion de minorité prendra corps dans les instruments juridiques. Dans ce domaine sans doute plus qu'ailleurs, la clarté d'une notion conditionne sa justiciabilité. 